\title{
Impact of Diabetes Mellitus on Radial and Ulnar Arterial Vasoreactivity after Radial Artery Cannulation: A Randomized Controlled trial
}

\author{
Eun Jung Kim ${ }^{1,2}$, Sarah Soh ${ }^{1,2}$, So Yeon Kim ${ }^{1,2}$, Hae Keum Kil1,2, Jae Hoon Lee ${ }^{1,2}$, Jeong Min Kim,2, Tae \\ Whan Kim¹, and Bon-Nyeo Koo ${ }^{1,2} \bowtie$ \\ 1. Department of Anesthesiology and Pain Medicine, Yonsei University College of Medicine, Seoul, Republic of Korea; \\ 2. Anesthesia and Pain Research Institute, Yonsei University College of Medicine, Seoul, Republic of Korea.
}

$\square$ Corresponding author: Bon-Nyeo Koo, MD, PhD. Department of Anesthesiology and Pain Medicine, Anesthesia and Pain Research Institute, Yonsei University College of Medicine, 50-1 Yonsei-ro, Seodaemun-gu, Seoul 120-752, Republic of Korea. Office Phone: +82-2-2228-8513 Fax: +82-2-2227-7897 E-mail: KOOBN@yuhs.ac.

(C) Ivyspring International Publisher. Reproduction is permitted for personal, noncommercial use, provided that the article is in whole, unmodified, and properly cited. See http://ivyspring.com/terms for terms and conditions.

Received: 2016.04.29; Accepted: 2016.07.27; Published: 2016.08.12

\begin{abstract}
Background: Endothelial dysfunction associated with diabetes mellitus (DM) may influence arterial vasoreactivity after arterial stimulus, such as cannulation, and cause changes in diameter and blood flow. Despite the frequent use of arterial cannulation during anesthesia and critical care, little information is available regarding vasoreactivity of the radial and ulnar arteries and its influence on underlying DM.

Methods: Forty non-DM and 40 DM patients, who required arterial cannulation during general anesthesia, were enrolled. Using duplex Doppler ultrasonography, we measured the patients' arterial diameter, peak systolic velocity, end-diastolic velocity, resistance index, and mean volume flow of both arteries at five different time points.

Results: After radial artery cannulation, ulnar arterial diameter and blood flow did not significantly increase in DM group, as they did in non-DM group. Ulnar arterial resistance index significantly increased in both groups, but the degree of decrease in DM group was significantly less than non-DM.

Conclusion: Ulnar artery's ability to increase blood flow for compensating the sudden reduction of radial arterial flow in DM patients was significantly less than that in non-DM patients under general anesthesia. Such attenuated vasoreactivity of ulnar artery to compensate the reduced radial arterial flow may have to be considered in radial arterial cannulation for DM patients.
\end{abstract}

Key words: catheterization; radial artery; ulnar artery; Diabetes mellitus; ultrasonography, doppler, duplex.

\section{Introduction}

Arterial cannulation is often required during anesthesia and critical care and provides valuable hemodynamic information and convenient access to arterial blood sampling without causing additional patient discomfort. [1,2] The radial artery is a common site for such invasive cannulation during major surgery due to its anatomical accessibility, ease of cannulation, and relatively low rate of complications because of high collaterality from the neighboring ulnar artery. $[3,4]$

Vasoreactivity is affected by hypertension, hypercholesterolemia, hyperglycemia, and diabetes mellitus (DM).[5] DM is associated with endothelial dysfunction, enhanced contractile response, and impaired vasorelaxation in response to clinically relevant vasoconstrictors and external stimuli, such as arterial cannulation.[6,7] The condition also causes arterial rigidity and increases peripheral vascular 
resistance, resulting in reduced blood flow, even when individuals have no apparent peripheral artery disease.[8] Although many studies have explored changes in radial artery vasoreactivity due to external stimuli and underlying conditions such as DM, limited information is available regarding $\mathrm{DM}^{\prime} \mathrm{s}$ effects on the ulnar artery after cannulation. Thus, to determine whether DM alters ulnar artery's vasoreactivity to compensate any reduction of radial arterial flow, we evaluated and compared serial Doppler patterns of ulnar artery as well as radial artery after radial artery cannulation in patients with and without DM under general anesthesia.

\section{Materials and Methods}

The human study protocol was approved by the Institutional Review Board of Severance Hospital, Yonsei University Health System (IRB\# 4-2013-0209) and was registered at http://Clinicaltrials.gov (registration \# NCT01897857). We obtained written informed consent from every patient in our study, which included 40 non-DM patients and $40 \mathrm{DM}$ patients aged 20 to 60 years. All patients were classified as either physical status III or IV according to the American Society of Anesthesiologists and were scheduled for elective kidney transplantation which was to be performed under general anesthesia. Every patient required arterial cannulation for continuous pressure monitoring during anesthesia and surgery. We excluded patients with known coagulopathy, signs of inflammation at the intended cannulation site, insufficient compensatory blood flow from the ulnar artery, or cannulation at an alternate site due to previous failed cannulation attempts.

The wrist joint of each patient was extended up to $45^{\circ}$ on a wrist board as described in a study by
Mizukoshi et al. [9] We selected the cannulation position where the most prominent radial artery pulsation around the radial styloid process occurred. Using B-mode ultrasound, we placed the probe axially $1 \mathrm{~cm}$ proximal to the marked point to measure the anterior-posterior internal diameter of the radial and ulnar arteries. We then perpendicularly placed the probe to obtain a circular image of the artery (Figure 1A, 2A). We measured the peak systolic velocity (PSV), end-diastolic velocity (EDV), resistance index (RI, RI = [PSV-EDV]/PSV), and mean volume flow (MVF) directly from a longitudinal duplex Doppler spectral waveform with an angle correction of $60^{\circ}$ (Figure 1B, 2B). Vessel compression was minimized using a conductive gel with decreased pressure on the probe. We collected transverse sections of the radial artery proximal to the presumed puncture site to obtain maximal diameter measurements and clear visualization of the arteries' internal lumen. Every image was acquired in triplicate.

Measurements were taken at five different time points: before anesthesia (T0); 5 min after anesthesia but before cannulation (T1); immediately after radial artery cannulation (T2); 20 min after catheter removal (T3); and $24 \mathrm{~h}$ after catheter removal (T4). At each time point, we measured hemodynamic parameters three times consecutively and calculated the mean values. When the radial artery diameter decreased by more than $20 \%$ of the pre-cannulation diameter, we noted cannulation-induced vasospasm. [10] Ultrasonographic measurements were obtained using a LOGIQe ${ }^{\mathrm{TM}}$ instrument (GE Healthcare, Wauwatosa, WI, USA) with a linear probe (12L-RS, $8-13 \mathrm{MHz}$ ) by a single experienced operator.

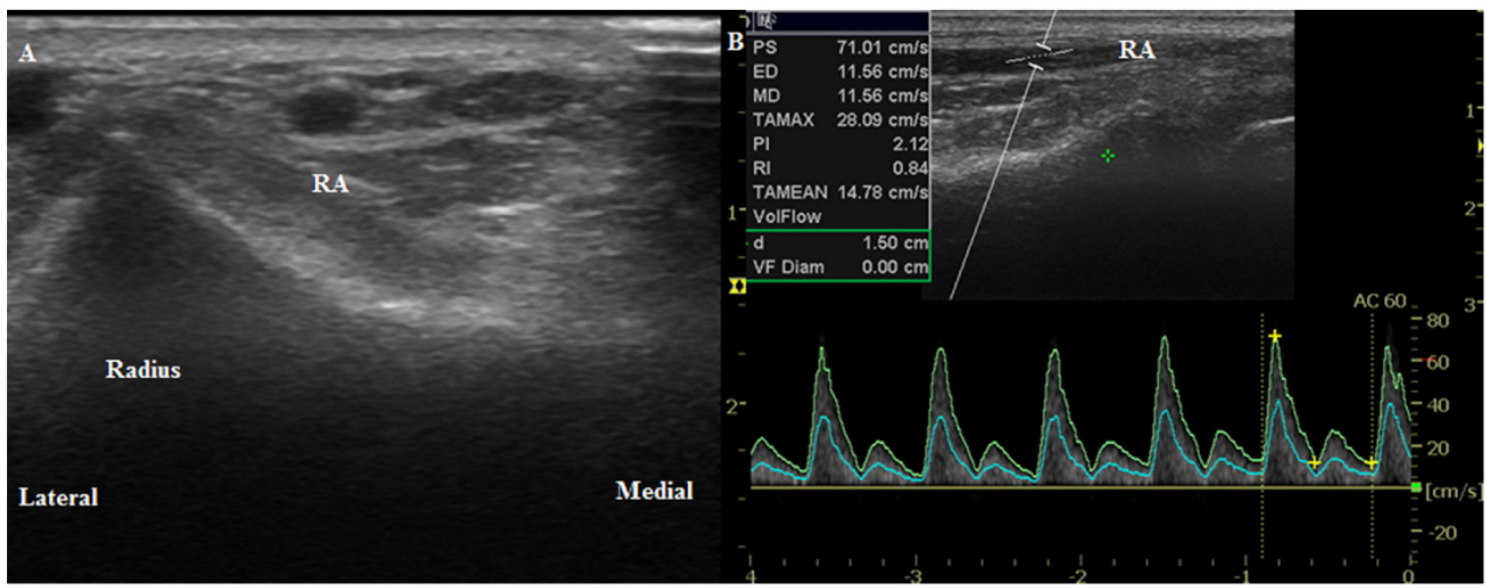

Figure 1. Ultrasound images of the radial artery. (A) Transverse circular image of the radial artery before anesthesia induction (T0). (B) Longitudinal duplex Dopppler spectral waveform with an angle correction of $60^{\circ}$. RA, radial artery. 


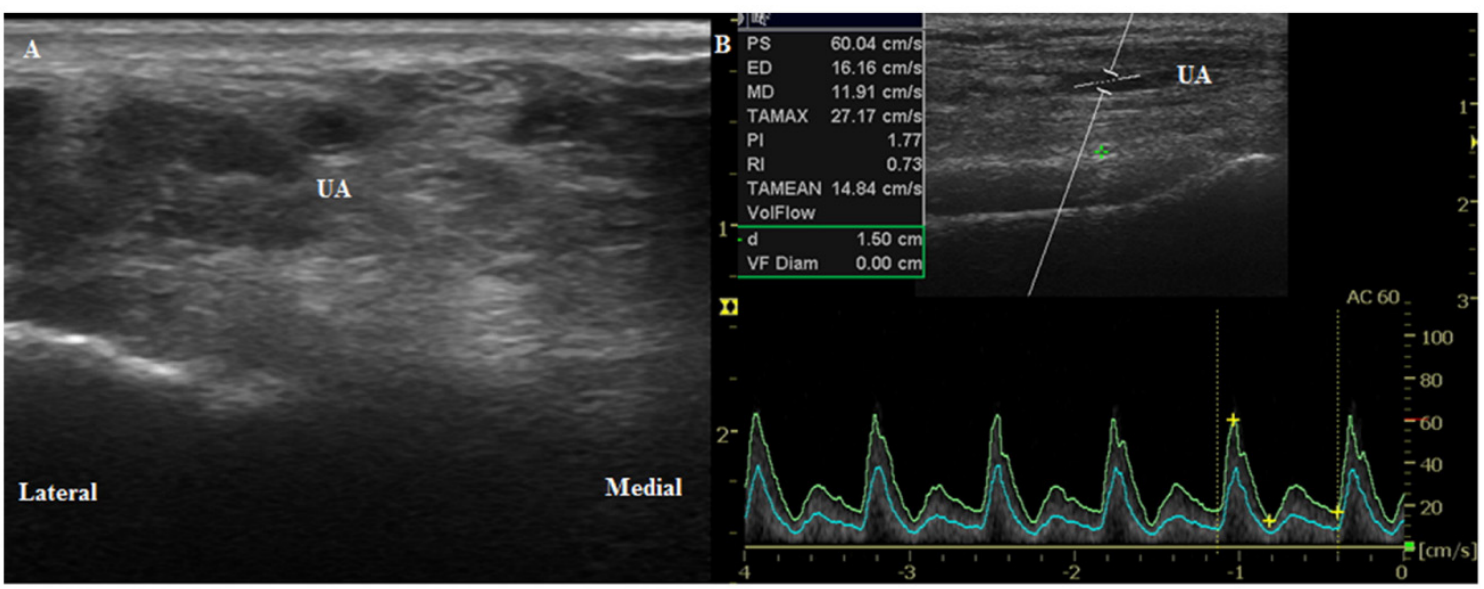

Figure 2. Ultrasound images of the ulnar artery. (A) Transverse circular image of the artery before anesthesia induction (T0). (B) Longitudinal duplex Dopppler spectral waveform with an angle correction of $60^{\circ}$. UA, ulnar artery.

General anesthesia was induced with $0.5-1$ $\mu \mathrm{g} / \mathrm{kg}$ remifentanil and $1.5-2 \mathrm{mg} / \mathrm{kg}$ propofol, and tracheal intubation was performed after administration of $0.8 \mathrm{mg} / \mathrm{kg}$ rocuronium bromide. Anesthesia was maintained with 0.05-0.15 $\mu \mathrm{g} / \mathrm{kg} / \mathrm{min}$ continuous infusion of remifentanil and $5.0-5.5 \%$ end-tidal desflurane in $50 \% \mathrm{O}_{2} /$ air. After successful intubation and completion of the Doppler measurement (T1), radial artery cannulation was performed by a single designated anesthesiologist using a uniform 20-G cannula (Biosafety IV Catheter, $1.1 \times 48 \mathrm{~mm}$, flow $55 \mathrm{ml} / \mathrm{min}$ (Sewon Medical Co., Cheonan, Korea)) via the palpation-guided simple catheter over needle technique, of which designated anesthesiologist was more proficient at, compared to rather unaccustomed ultrasound-guided method. The cannula was then securely fixed using transparent adhesive film. The number of cannulation attempts was limited to two trials; patients who experienced two failed cannulation attempts were excluded from the study. We minimized the frequency and amount of manual flushing of the arterial cannula with non-heparinized fluid and avoided unnecessary manipulation or stimulation of the arterial walls and cannula.

\section{Statistical analysis}

Sample size calculations were based on arterial diameter changes reported by a previous study, [11] in which the standard deviation of the radial artery diameter was $0.45 \mathrm{~mm}$. Therefore, 36 subjects in each group (DM or non-DM) were required to detect a $0.3-\mathrm{mm}$ difference in the radial artery diameters between the two groups at post-cannulation period using a two-sided a of $5 \%$ and $\beta$ power of $80 \%$. To account for a potential $10 \%$ dropout rate in each group, we included 40 patients per group. Statistical analysis of patient data was performed using PASW
Statistics 20 (SPSS Inc., Chicago, IL, USA) and SAS software version 9.2 (SAS Institute Inc., Cary, NC, USA). Patient characteristics were analyzed using an independent t-test, and categorical variables were evaluated using a Chi-squared test or Fisher's exact test when appropriate. Values are reported as means \pm standard deviation (SD), median (interquartile range, IQR) or as numbers of patients (\%). Comparison of changes in hemodynamic characteristics to their corresponding baseline values were performed with linear mixed models with post-hoc tests using Bonferroni correction. Nonparametric repeated measure variables were analyzed using the Friedman test or Mann-Whitney $U$ test. A value of $P<0.05$ indicated statistical significance.

\section{Results}

Forty non-DM and 40 DM patients were recruited between July 2013 and November 2015, all of whom completed the study. Demographic and baseline clinical characteristics of 40 non-DM and 40 DM human patients are presented in Table 1. Patients with DM showed a higher incidence of accompanied hypertension than non-DM patients $(98 \%$ vs. $78 \%$, respectively; $P=0.021$ ) and had, on average, experienced $\mathrm{DM}$ for 11 years. We observed no significant intergroup differences with respect to demographics, presence of atherosclerosis, medications, surgery-related data, or arterial cannulation-related clinical variables. Despite the increased incidence of hypertension in DM patients, mean blood pressures (MBP) were comparable between the two groups (Table 2). Such likewise were also noted at 2 days before and after surgery during which the effect of surgery-related anxiety or postoperative pain had been minimized (non-DM vs. DM; 91 vs. $91 \mathrm{mmHg}, P=0.973$ at 2 days before surgery; 99 vs. $98 \mathrm{mmHg}, P=0.571$ at 2 days after 
surgery). In addition, baseline radial and ulnar values of internal diameter, PSV, EDV, RI, and MVF did not differ significantly between non-DM and DM patients (Figure 3, 4).

Radial artery diameter increased in non-DM patients after anesthesia and persisted after radial artery cannulation (Figure 3A). However, the diameter decreased immediately after catheter removal, resulting in similar diameters between the two groups, although a greater radial diameter was again seen in non-DM patients $24 \mathrm{~h}$ after catheter removal. Radial artery MVF, determined by timeaveraged mean velocity and internal diameter of the corresponding artery, in non-DM patients more than doubled after anesthesia and cannulation, returned to baseline value after catheter removal in radial artery, and redoubled $24 \mathrm{~h}$ after catheter removal (Figure 3B). In contrast, although we observed increased radial artery MVF in DM patients, the values were not significantly different from baseline data. The RI of non-DM patients decreased by $20 \%$ and $16 \%$ after anesthesia and cannulation, respectively (Figure 3C), while DM patients experienced a more modest decrease of $14 \%$ after anesthesia and $16 \%$ after cannulation.
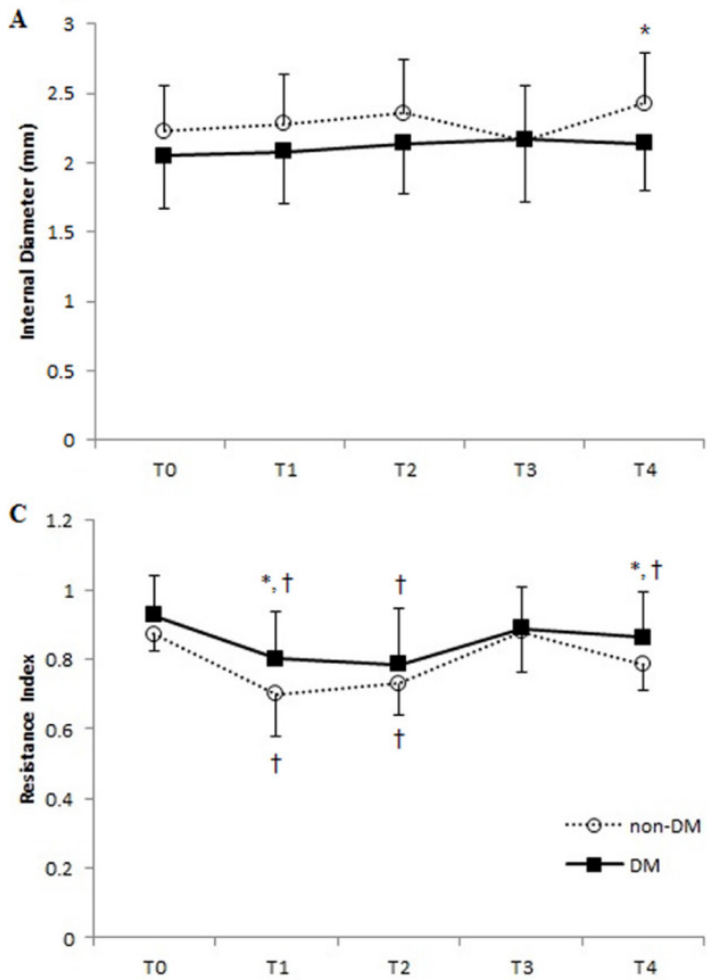

Table 1. Patient characteristics.

\begin{tabular}{|c|c|c|c|}
\hline & Non-DM $(n=40)$ & $\mathrm{DM}(\mathrm{n}=40)$ & $P$-value \\
\hline Age (years) & $42 \pm 11$ & $53 \pm 8$ & 0.084 \\
\hline Female & $19(48)$ & $13(33)$ & 0.149 \\
\hline Height $(\mathrm{cm})$ & $165 \pm 9$ & $165 \pm 8$ & 0.975 \\
\hline Weight $(\mathrm{kg})$ & $60 \pm 12$ & $64 \pm 12$ & 0.165 \\
\hline Hypertension & $31(78)$ & $39(98)$ & 0.021 \\
\hline Atherosclerosis & $6(15)$ & $7(18)$ & 0.801 \\
\hline \multicolumn{4}{|l|}{ Medications } \\
\hline Nitrates & $1(3)$ & $1(3)$ & 0.986 \\
\hline$\beta$-blockers & $20(50)$ & $14(35)$ & 0.212 \\
\hline Calcium antagonists & $22(55)$ & $16(40)$ & 0.221 \\
\hline ACE inhibitors & $2(5)$ & $2(5)$ & 0.980 \\
\hline $\mathrm{ARB}$ & $15(38)$ & $17(43)$ & 0.588 \\
\hline Smoking & $10(25)$ & $17(43)$ & 0.118 \\
\hline ASA 3:4 & $36: 4$ & $34: 6$ & 0.356 \\
\hline Cholesterol (mg/dL) & $150 \pm 43$ & $154 \pm 39$ & 0.666 \\
\hline $\mathrm{LDL}(\mathrm{mg} / \mathrm{dL})$ & $81 \pm 29$ & $77 \pm 34$ & 0.624 \\
\hline $\operatorname{HDL}(\mathrm{mg} / \mathrm{dL})$ & $41 \pm 13$ & $45 \pm 23$ & 0.282 \\
\hline $\mathrm{TG}(\mathrm{mg} / \mathrm{dL})$ & $138 \pm 108$ & $127 \pm 75$ & 0.593 \\
\hline Glucose $(\mathrm{mg} / \mathrm{dL})$ & $108 \pm 22$ & $152 \pm 46$ & $<0.001$ \\
\hline HbA1c (\%) & $5.3 \pm 0.4$ & $6.5 \pm 0.9$ & $<0.001$ \\
\hline $\begin{array}{l}\text { Number of arterial blood } \\
\text { sampling }\end{array}$ & $4(4-6)$ & $4(4-5)$ & 0.254 \\
\hline Irrigation fluid (mL) & $55 \pm 16$ & $51 \pm 19$ & 0.332 \\
\hline Cannulation time (min) & $326 \pm 38$ & $328 \pm 55$ & 0.814 \\
\hline Op time (min) & $262 \pm 40$ & $255 \pm 58$ & 0.536 \\
\hline Complication* & $18(45)$ & $10(25)$ & 0.224 \\
\hline
\end{tabular}

Values expressed as means $\pm \mathrm{SD}$, numbers of patients (\%) or medians (IQR). ${ }^{*}$ Complication indicates any event of vasospasm or thrombosis resulting from radial artery cannulation.

ACE inhibitor, angiotensin converting enzyme inhibitor; ARB, angiotensin II receptor blockers; ASA, American Society of Anesthesiologists Classification; LDL low-density lipoprotein; HDL, high-density lipoprotein; TG, triglyceride; HbA1c, hemoglobin A1c.

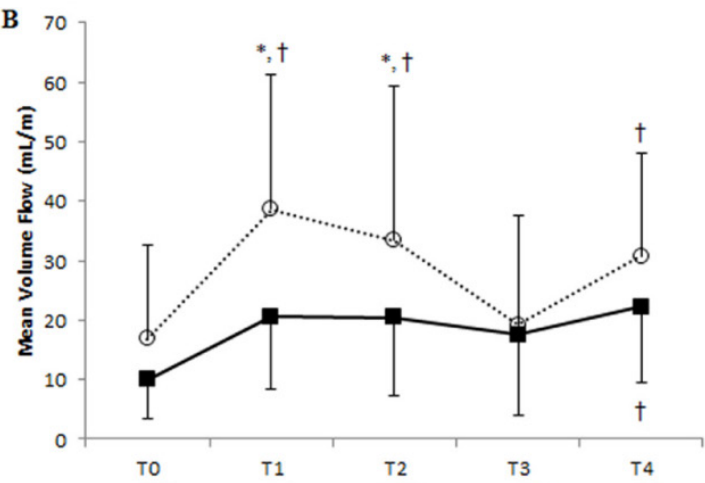

Figure 3. Vascular parameters of the radial artery at each time point in DM and non-DM groups. Means of $(A)$ internal diameter, (B) mean volume flow, and (C) resistance index are shown. Error bars represent SD. a, DM group; $\circ$, non-DM group. T0, before anesthesia; T1, 5 min after anesthesia and before cannulation; T2, immediately after radial artery cannulation; T3, 20 min after catheter removal; T4, 24 hours after catheter removal. $* P<0.05$ of DM vs. non-DM group at each time point; $\nmid P<0.05$ of T0 within each group. 

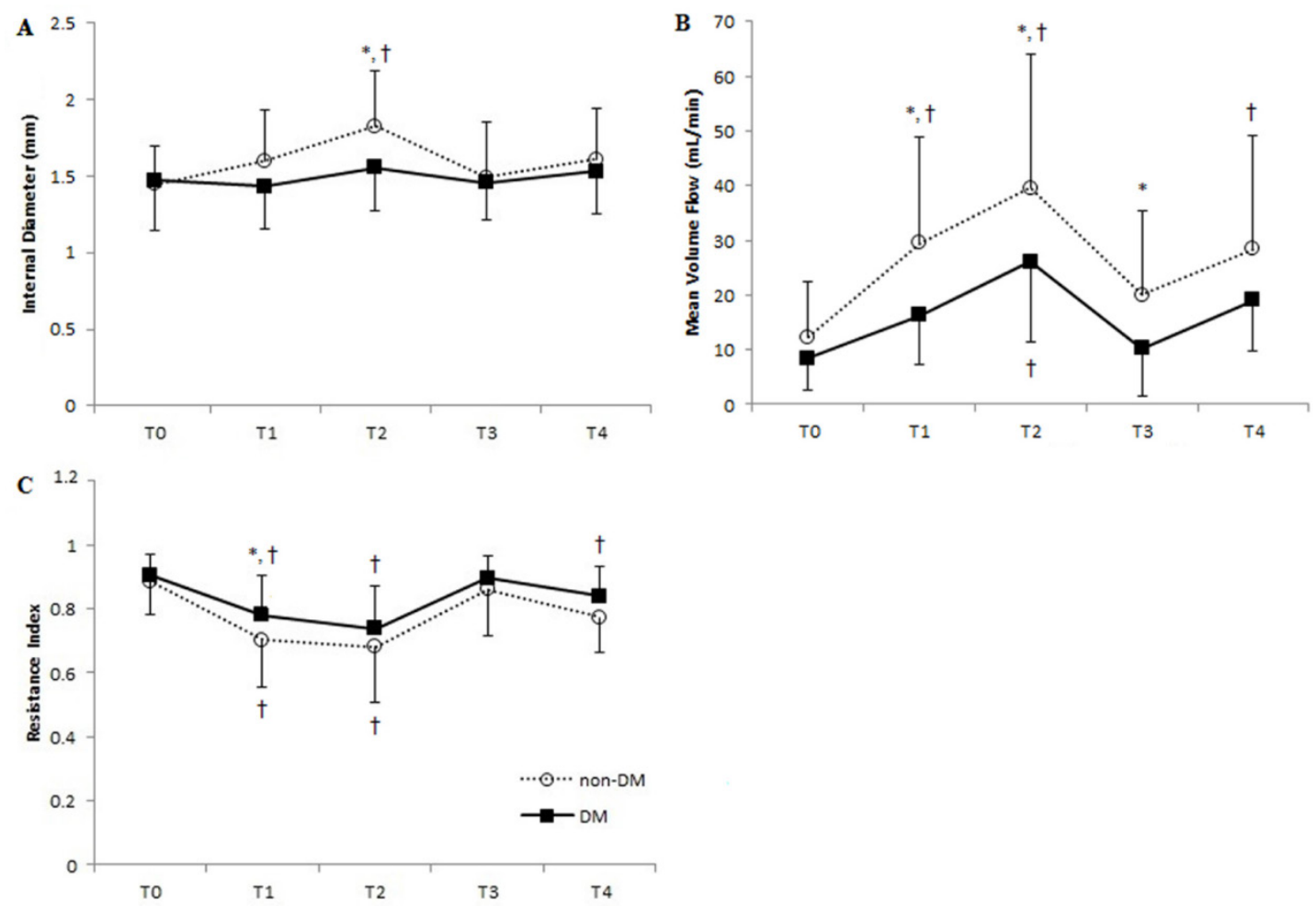

Figure 4. Vascular parameters of the ulnar artery at each time point in DM and non-DM patients. Means of $(A)$ internal diameter, (B) mean volume flow, and (C) resistance index are shown. Error bars represent SD. .., DM group; $\circ$, non-DM group. T0, before anesthesia; T1, 5 min after anesthesia and before cannulation; T2, immediately after radial artery cannulation; T3, 20 min after catheter removal; T4, 24 hours after catheter removal. $* P<0.05$ of DM vs. non-DM group at each time point; $\dagger P<0.05$ of TO within each group.

Table 2. Hemodynamics at each time point.

\begin{tabular}{llll}
\hline & $\begin{array}{l}\text { Non-DM } \\
(\mathrm{n}=40)\end{array}$ & $\mathrm{DM}(\mathrm{n}=40)$ & $P$-value \\
\hline $\begin{array}{l}\text { Mean blood pressure } \\
\text { (mmHg) }\end{array}$ & & & \\
T0 & $108 \pm 17$ & $110 \pm 17$ & 0.635 \\
T1 & $98 \pm 14$ & $99 \pm 13$ & 0.815 \\
T2 & $92 \pm 13$ & $92 \pm 19$ & 0.992 \\
T3 & $104 \pm 11$ & $99 \pm 12$ & 0.055 \\
T4 & $107 \pm 11$ & $103 \pm 11$ & 0.070 \\
Heart rate (beats/min) & & & \\
T0 & $80 \pm 115$ & $78 \pm 12$ & 0.591 \\
T1 & $81 \pm 17$ & $76 \pm 11$ & 0.142 \\
T2 & $79 \pm 17$ & $75 \pm 13$ & 0.272 \\
T3 & $84 \pm 11$ & $80 \pm 13$ & 0.100 \\
T4 & $77 \pm 12$ & $82 \pm 13$ & 0.054 \\
\hline
\end{tabular}

Values are means $\pm \mathrm{SD}$. T0, before anesthesia; T1, 5 min after anesthesia and before cannulation; T2, immediately after radial artery cannulation; T3, 20 min after catheter removal; T4, 24 hours after catheter removal.

Ulnar artery MVF in non-DM patients was 3.4 times higher after anesthesia and cannulation compared to baseline values. We also observed a $29 \%$ increase in ulnar artery diameter in these patients due to compensatory dynamics from the interrupted radial artery blood flow resulting from cannulation (Figures 4A, 4B). We saw similar, but more subtle, trends in the DM patients, in which compensatory changes increased ulnar artery MVF to 2.7 times higher than baseline and caused a 5.6\% increase in internal diameter. Ulnar artery RI after anesthesia significantly decreased in both non-DM and DM patients, who experienced $21 \%$ and $14 \%$ reduction of baseline values, respectively. However, we saw no significant difference in RI between the two groups with respect to cannulation and catheter removal (Figure 4C). No patients reported hematoma formation, thrombosis, cannulation-related pain, or sensory changes before hospital discharge.

\section{Discussion}

Our results demonstrate the impact of underlying DM on arterial vasoreactivity after simple catheter cannulation during surgery. Specifically, we noted that patients with DM were temporally unsuccessful in showing adaptive vasoreactivity of the radial and ulnar arteries after radial artery cannulation under general anesthesia. Fortunately, the internal diameter and blood flow of both arteries returned to near-baseline levels in all patients $24 \mathrm{~h}$ after removal of their radial artery catheter.

Due to easy accessibility and low rate of associated complications, the radial artery is favored in various clinical procedures, such as for a bypass conduit during coronary artery bypass graft (CABG) 
surgery, $[12,13]$ as the site of cardiac catheterization in coronary angiography,[14] and as the route of cannulation for continuous monitoring of arterial pressure.[2] In addition, the artery's vascular response to external stimuli (vasoreactivity) has been investigated extensively.[13,15-17] Radial artery harvest has shown mild reduction in digital perfusion and complementary increase in ulnar artery diameter and flow velocity with no clinical sequelae or compromised hand function.[18,19] After catheterization for coronary angiography access, the artery may exhibit reduced lumen density due to wall injuries after cannulation.[20,21]

In contrast to the immense amount of information available regarding radial artery vasoreactivity with respect to vascular surgery and coronary intervention, very few studies have explored changes in radial artery related to cannulation access for real-time blood pressure monitoring. [10,22] Similarly, although DM strongly influences vasoreactivity,[23,24] its specific impact on vasoreactivity after simple arterial cannulation for invasive blood pressure monitoring is not well-studied. The procedure is widely adopted, yet still carries a high risk of complications, especially in patients with atherosclerosis-related risk factors. The frequency of previously reported vascular complications after radial artery cannulation ranges from $1.5 \%$ to $88 \%$, [2] which widely varies from temporary spasm to distal limb ischemia. Other possible complications include bleeding (0.5\%), hematoma $(14.4 \%)$, pseudoaneurysm (0.09\%), infection $(0.72 \%)$, and sepsis $(0.13 \%)$. [2,3] In diabetic patients, the incidence of complications is higher as evidenced by histomorphological and molecular characterization of the arteries. $[13,25]$

Hyperglycemia influences vasoreactivity and vessel integrity, which are also affected by the balance between eNOS (endothelial NOS) and endothelin. $[13,26]$ Endothelium-associated eNOS can generate nitric oxide (NO), which regulates vascular tonus according to blood flow demand, [27] while the vasoactive peptide endothelin contributes to vasoconstriction in vascular smooth muscle cells. Moreover, insulin resistance found in patients with $\mathrm{DM}$ impairs $\mathrm{NO}$ synthesis and trapping of $\mathrm{NO}$ reactive oxygen species which can decrease $\mathrm{NO}$ bioavailability.[28] Vasoreactivity and the consequent alteration of vessel diameter are known collectively as arterial remodeling.[29] In studies focused on such vascular response, patients with hyperglycemia and DM fail to exert compensational arterial changes after exposure to an external stimulus, $[23,28,30]$ and may deter rapid recovery of patients.[6,31] Similarly, we observed that DM patients showed little or no changes in radial artery internal diameter, blood flow, or RI compared to baseline values, while significant changes in such arterial hemodynamics of non-DM patients occurred probably due to the changes in vasoactive balance, sympathetic tone, and external stimuli throughout perioperative period. The ulnar artery, which often provides active compensation after neighboring radial artery cannulation, also showed only subtle changes in its arterial hemodynamics among DM patients, indicating less compensation for decreased blood flow from damaged radial artery. Interestingly, we also observed some changes in vascular hemodynamics before the arterial cannulation. Per our study hypothesis, which focused on radial artery cannulation and consequential changes in the ulnar artery, we observed significant changes in arterial diameter, blood flow, and RI, possibly due to reduced sympathetic tone after anesthesia. Moreover, as our study had focused on the changes in vascular reactivity followed by relatively lesser dealt simple arterial cannulation, such distinguishing changes in vasoreactivity according to underlying DM can also be applied in better understanding of patients' outcomes in other surgical procedures, such as CABG, and for cardiac catheterization in coronary angiography.

Our study has a few limitations. First, in order to avoid unnecessary arterial cannulation and only to recruit those who actually require arterial cannulation for invasive blood pressure monitoring during surgery, this study enrolled those who were scheduled for kidney transplantation. Such group of patients could inevitably affect the vasoreactivity of the vessels due to the arteriopathy related to primary renal disease. Second, intergroup differences in underlying hypertension and DM prevalence could have affected endothelial characteristics, as these conditions accelerate DM-associated microvascular and macrovascular complications. Although neither DM nor non-DM patients showed significant differences with respect to atherosclerosis incidence, the possibility that non-DM patients may have other atherosclerosis-related systemic diseases should not be overlooked. Such patient characteristics could possibly influence endothelial dysfunction in parallel to DM. Lastly, we could increase accuracy of the ultrasound measurements only so much, although we maximized its utility by taking multiple measurements by one well-trained sonographer for all scans. Alternative high-accuracy methods, such as arteriography, plethysmography, and flow-mediated dilation, are available but do not provide the same ease and accessibility as sonography.

In conclusion, diabetic patients lack ulnar 
artery's vasoreactivity to compensate for sudden changes in radial arterial flow following radial artery cannulation under general anesthesia.

\section{Abbreviations}

DM: Diabetes mellitus; PSV: Peak systolic velocity; EDV: End-diastolic velocity; RI: Resistance index; MVF: Mean volume flow; SD: Standard deviation; IQR: Interquartile range; MBP: Mean blood pressures; eNOS: endothelial NOS; NO: Nitric oxide.

\section{Acknowledgements}

This work was supported by the National Research Foundation of Korea (NRF) grant funded by the Korea government (MSIP) (No. 2014R1A2A2A01007289)

\section{Competing interests}

The authors declare that they have no competing interests.

\section{References}

1. Miller AG, Bardin AJ. Review of Ultrasound-Guided Radial Artery Catheter Placement. Resp Care. 2016; 61(3): 383-8.

2. Brzezinski M, Luisetti $T$, London MJ. Radial artery cannulation: a comprehensive review of recent anatomic and physiologic investigations. Anesth Analg. 2009; 109(6): 1763-81.

3. Scheer B, Perel A, Pfeiffer UJ. Clinical review: complications and risk factors of peripheral arterial catheters used for haemodynamic monitoring in anaesthesia and intensive care medicine. Crit Care. 2002; 6(3): 199-204.

4. Hall JJ, Arnold AM, Valentine RP, McCready RA, Mick MJ. Ultrasound imaging of the radial artery following its use for cardiac catheterization. Am J Cardiol. 1996; 77(1): 108-9.

5. Pennathur S, Heinecke JW. Oxidative stress and endothelial dysfunction in vascular disease. Curr Diabetes Rep. 2007; 7(4): 257-64.

6. Le Guillou V, Tamion F, Jouet I, Richard V, Mulder P, Bessou JP, et al. Mesenteric endothelial dysfunction in a cardiopulmonary bypass rat model: the effect of diabetes. Diabetes Vasc Dis Re. 2012; 9(4): 270-9.

7. Roffi M, Angiolillo DJ, Kappetein AP. Current concepts on coronary revascularization in diabetic patients. Eur Heart J. 2011; 32(22): 2748-57.

8. Suzuki E, Kashiwagi A, Nishio Y, Egawa K, Shimizu S, Maegawa H, et al. Increased arterial wall stiffness limits flow volume in the lower extremities in type 2 diabetic patients. Diabetes Care. 2001; 24(12): 2107-14.

9. Mizukoshi K, Shibasaki M, Amaya F, Hirayama T, Shimizu F, Hosokawa K, et al. Ultrasound evidence of the optimal wrist position for radial artery cannulation. Can J Anaesth. 2009; 56(6): 427-31.

10. Kim SY, Lee JS, Kim WO, Sun JM, Kwon MK, Kil HK. Evaluation of radial and ulnar blood flow after radial artery cannulation with 20- and 22-gauge cannulae using duplex Doppler ultrasound. Anaesthesia. 2012; 67(10): 1138-45.

11. Sanmartin M, Goicolea J, Ocaranza R, Cuevas D, Calvo F. Vasoreactivity of the radial artery after transradial catheterization. J Invasive Cardiol. 2004; 16(11): 635-8.

12. Desai ND, Cohen EA, Naylor CD, Fremes SE. A randomized comparison of radial-artery and saphenous-vein coronary bypass grafts. New Engl J Med. 2004; 351(22): 2302-9.

13. Zou L, Chen X, Chen W, Li L, Huang F, Xiang F. Comparative study on the histomorphology and molecular biology of radial artery conduits in patients with diabetes mellitus who underwent coronary bypass surgery. Diabetes Vasc Dis Re. 2013; 10(3): 208-15.

14. Valgimigli M, Gagnor A, Calabró P, Frigoli E, Leonardi S, Zaro T, et al. Radial versus femoral access in patients with acute coronary syndromes undergoing invasive management: a randomised multicentre trial. Lancet. 2015; 385(9986): 2465-76.

15. Sellke FW, Boyle EM, Verrier ED. Endothelial cell injury in cardiovascular surgery: the pathophysiology of vasomotor dysfunction. Ann Thorac Surg. 1996; 62(4): 1222-8

16. Medalion B, Tobar A, Yosibash Z, Stamler A, Sharoni E, Snir E, et al. Vasoreactivity and histology of the radial artery: comparison of open versus endoscopic approaches. Eur J Cardio-Thorac. 2008; 34(4): 845-9.

17. Chester AH, Amrani M, Borland JA. Vascular biology of the radial artery. Curr Opin Cardiol. 1998; 13(6): 447-52.
18. Brodman RF, Hirsh LE, Frame R. Effect of radial artery harvest on collateral forearm blood flow and digital perfusion. J Thorac Cardiov Sur. 2002; 123(3): 512-6.

19. Işık M, Yüksek T, Dereli Y, Görmüş N, Durgut K, Koç O. [Evaluation of post-operative flow and diameter changes in brachial and ulnar arteries in coronary artery bypass surgery patients in which the radial artery is used as graft]. Turk Kardiyol Dern Ars. 2015; 43(7): 630-6.

20. Ruiz Salmerón RJ, Mora R, Vélez Gimón M, Ortiz J, Fernández C, Vidal B, et al. [Radial artery spasm in transradial cardiac catheterization. Assessment of factors related to its occurrence, and of its consequences during follow-up]. Rev Esp Cardiol. 2005; 58(5): 504-11.

21. Costa F, van Leeuwen MA, Daemen J, Diletti R, Kauer F, van Geuns R, et al. The Rotterdam Radial Access Research: Ultrasound-Based Radial Artery Evaluation for Diagnostic and Therapeutic Coronary Procedures. Circ Cardiovasc Interv. 2016; 9(2): e003129.

22. Eker HE, Tuzuner A, Yilmaz AA, Alanoglu Z, Ates Y. The impact of two arterial catheters, different in diameter and length, on postcannulation radial artery diameter, blood flow, and occlusion in atherosclerotic patients. J Anesth. 2009; 23(3): 347-52.

23. Choudhary BP, Antoniades C, Brading AF, Galione A, Channon K, Taggart DP. Diabetes mellitus as a predictor for radial artery vasoreactivity in patients undergoing coronary artery bypass grafting. J Am Coll Cardiol. 2007; 50(11): 1047-53.

24. Tajima Y, Suzuki E, Saito J, Murase H, Horikawa Y, Takeda J. Elevated plasma B-type natriuretic peptide concentration and resistive index, but not decreased aortic distensibility, associate with impaired blood flow at popliteal artery in type 2 diabetic patients. Endocr J. 2015; 62(6): 503-11.

25. Hammoud T, Tanguay JF, Bourassa MG. Management of coronary artery disease: therapeutic options in patients with diabetes. J Am Coll Cardiol. 2000; 36(2): 355-65.

26. Brownlee M. Biochemistry and molecular cell biology of diabetic complications. Nature. 2001; 414(6865): 813-20.

27. Lüscher TF, Diederich D, Siebenmann R, Lehmann K, Stulz P, von Segesser L, et al. Difference between endothelium-dependent relaxation in arterial and in venous coronary bypass grafts. New Engl J Med. 1988; 319(8): 462-7.

28. Soran O. Percutaneous versus surgical interventions for coronary artery disease in those with diabetes mellitus. Curr Cardiol Rep. 2013; 15(1):323.

29. Manabe S, Sunamori M. Radial artery graft for coronary artery bypass surgery: biological characteristics and clinical outcome. J Cardiac Surg. 2006; 21(1): 102-14.

30. Lehle K, Preuner JG, Vogt A, Rupprecht L, Keyser A, Kobuch R, et al. Endothelial cell dysfunction after coronary artery bypass grafting with extracorporeal circulation in patients with type 2 diabetes mellitus. Eur J Cardio-Thorac. 2007; 32(4): 611-6.

31. Jackson CV, Carrier GO. Influence of short-term experimental diabetes on blood pressure and heart rate in response to norepinephrine and angiotensin II in the conscious rat. J Cardiovasc Pharm. 1983; 5(2): 260-5 